\title{
Extending Fourier transformations to Hamilton's quaternions and Clifford's geometric algebras
}

\author{
Eckhard Hitzer
}

Department of Material Science, International Christian University, 181-8585 Japan

\begin{abstract}
We show how Fourier transformations can be extended to Hamilton's algebra of quaternions. This was initially motivated by applications in nuclear magnetic resonance and electric engineering. Followed by an ever wider range of applications in color image and signal processing. Hamilton's algebra of quaternions is only one example of the larger class of Clifford's geometric algebras, complete algebras encoding a vector space and all its subspace elements. We introduce how Fourier transformations are extended to Clifford algebras and applied in electromagnetism, and in the processing of images, color images, vector field and climate data.
\end{abstract}

Keywords: Clifford geometric algebra, quaternion Fourier transform, Clifford Fourier transform, Clifford Fourier-Mellin transform, Mulitvector wavepackets, Spacetime Fourier transform

PACS: AMS Subj. Class. 15A66, 42A38

There is the alternative operator exponential Clifford Fourier transform (CFT) approach, mainly pursued by the Clifford Analysis Group at the university of Ghent (Belgium) [2]. Our aim here is to provide an overview of research based on the holistic investigation [21] of real geometric square roots of -1 in Clifford algebras $C l(p, q)$ over real vector spaces $\mathbb{R}^{p, q}$. These algebras include real and complex numbers, quaternions, Pauli- and Dirac algebra, space time algebra, spinor algebra, Lie algebras, conformal geometric algebra and many more. The resulting CFTs are therefore perfectly tailored to work on functions valued in these algebras. In general the continuous manifolds of $\sqrt{-1}$ in $C l(p, q)$ consist of several conjugacy classes and their connected components. Simple examples are shown in Fig. 1.

A CFT analyzes scalar, vector and multivector signals in terms of sine and cosine waves with multivector coefficients. Basically, the imaginary unit $i \in \mathbb{C}$ in the transformation kernel $e^{i} \phi=\cos \phi+i \sin \phi$ is replaced by a $\sqrt{-1}$ in $\mathrm{Cl}(p, q)$. This produces a host of CFTs, an incomplete brief overview is sketched in Fig. 2, see also the historical overview in [2]. Additionally the $\sqrt{-1}$ in $C l(p, q)$ allow to construct further types of integral transformations, notably Clifford wavelets $[16,25]$.

Recently a rigorous effort was made in [5] to design a general geometric Fourier transform, that incorporates most of the previously known CFTs with the help of very general sets of left and right kernel factor products

$$
\mathscr{F}_{G F T}\{h\}(\omega)=\int_{\mathbb{R}^{p^{\prime}, q^{\prime}}} L(\mathbf{x}, \omega) h(\mathbf{x}) R(\mathbf{x}, \omega) d^{n^{\prime}} \mathbf{x}, \quad L(\mathbf{x}, \omega)=\prod_{s \in F_{L}} e^{-s(\mathbf{x}, \omega)},
$$

with $p^{\prime}+q^{\prime}=n^{\prime}, F_{L}=\left\{s_{1}(\mathbf{x}, \omega), \ldots, s_{L}(\mathbf{x}, \omega)\right\}$ a set of mappings $\mathbb{R}^{p^{\prime}, q^{\prime}} \times \mathbb{R}^{p^{\prime}, q^{\prime}} \rightarrow \mathscr{I}^{p, q}$ into the manifold of real multiples of $\sqrt{-1}$ in $C l(p, q) . R(\mathbf{x}, \omega)$ is defined similarly, and $h: \mathbb{R}^{p^{\prime}, q^{\prime}} \rightarrow C l(p, q)$ is the multivector signal function.

This clearly subsumes the CFT due to Sommen and Buelow [4]

$$
\mathscr{F}_{S B}\{h\}(\omega)=\int_{\mathbb{R}^{n}} h(\mathbf{x}) \prod_{k=1}^{n} e^{-2 \pi x_{k} \omega_{k} e_{k}} d^{n} \mathbf{x},
$$

where $\mathbf{x}, \omega \in \mathbb{R}^{n}$ with components $x_{k}, \omega_{k}$, and $\left\{e_{1}, \ldots e_{k}\right\}$ is an orthonormal basis of $\mathbb{R}^{0, n}, h: \mathbb{R}^{n} \rightarrow C l(0, n)$.

It is further possible [11] to only pick strictly mutually commuting sets of $\sqrt{-1}$ in $C l(p, q)$, e.g. $e_{1} e_{2}, e_{3} e_{4} \in C l(4,0)$ and construct CFTs with therefore commuting kernel factors in analogy to (2). Also contained in (1) is the color image CFT of [26]

$$
\mathscr{F}_{C I}\{h\}(\omega)=\int_{\mathbb{R}^{2}} e^{\frac{1}{2} \omega \cdot \mathbf{x} I_{4} B} e^{\frac{1}{2} \omega \cdot \mathbf{x} B} h(\mathbf{x}) e^{-\frac{1}{2} \omega \cdot \mathbf{x} B} e^{-\frac{1}{2} \omega \cdot \mathbf{x} I_{4} B} d^{2} \mathbf{x}
$$

where $B \in C l(4,0)$ is a bivector and $I_{4} B \in C l(4,0)$ its dual complementary bivector. It is especially useful for the introduction of efficient non-marginal generalized color image Fourier descriptors. 
The main type of CFT, which we will review here is the general two sided CFT [19] with only one kernel factor on each side

$$
\mathscr{F}^{f, g}\{h\}(\omega)=\int_{\mathbb{R}^{p^{\prime}, q^{\prime}}} e^{-f u(\mathbf{x}, \omega)} h(\mathbf{x}) e^{-g v(\mathbf{x}, \omega)} d^{n^{\prime}} \mathbf{x},
$$

with $f, g$ two $\sqrt{-1}$ in $C l(p, q), u, v: \mathbb{R}^{p^{\prime}, q^{\prime}} \times \mathbb{R}^{p^{\prime}, q^{\prime}} \rightarrow \mathbb{R}$ and often $\mathbb{R}^{p^{\prime}, q^{\prime}}=\mathbb{R}^{p, q}$. In the following we will discuss eleven transforms, which belong to this class of CFTs, see the lower half of Fig. 2.

One of the nowadays most widely applied CFTs is the quaternion Fourier transform (QFT) [14, 20]

$$
\mathscr{F}^{f, g}\{h\}(\omega)=\int_{\mathbb{R}^{2}} e^{-f x_{1} \omega_{1}} h(\mathbf{x}) e^{-g x_{2} \omega_{2}} d^{2} \mathbf{x},
$$

which also has variants were one of the left or right kernel factors is dropped, or both are placed together at the right or left side. It was first described by Ernst, et al, [10, pp. 307-308] (with $f=\boldsymbol{i}, g=\boldsymbol{j}$ ) for spectral analysis in twodimensional nuclear magnetic resonance, suggesting to use the QFT as a method to independently adjust phase angles with respect to two frequency variables in two-dimensional spectroscopy. Later Ell [8] independently formulated and explored the QFT for the analysis of linear time-invariant systems of PDEs. The QFT was further applied by Buelow, et al [3] for image, video and texture analysis, by Sangwine et al [27, 2] for color image analysis and analysis of non-stationary improper complex signals, vector image processing, and quaternion polar signal representations. It is possible to split every quaternion-valued signal and its QFT into two quasi-complex components [20], which allow the application of complex discretization and fast FT methods. The split can be generalized to the general CFT (4) [19] in the form

$$
x_{ \pm}=\frac{1}{2}(x \pm f x g), \quad x \in C l(p, q)
$$

In the case of quaternions the quaternion coefficient space $\mathbb{R}^{4}$ is thereby split into two steerable (by the choice of $f, g$ ) orthogonal two-dimensional planes [20]. For colors expressed by quaternions, these two planes become chrominance and luminance when $f=g=$ gray line [9].

Georgiev and Morais have modified the QFT to a quaternion Fourier Stieltjes transform [13].

$$
\mathscr{F}_{S t j}\left(\sigma^{1}, \sigma^{2}\right)=\int_{\mathbb{R}^{2}} e^{-f x_{1} \omega_{1}} d \sigma^{1}\left(x_{1}\right) d \sigma^{2}\left(x_{2}\right) e^{-g x_{2} \omega_{2}},
$$

with $f=-\boldsymbol{i}, g=-\boldsymbol{j}, \sigma^{k}: \mathbb{R} \rightarrow \mathbb{H},\left|\sigma^{k}\right| \leq \delta_{k}$ for real numbers $0<\delta_{k}<\infty, k=1,2$. Introducing polar coordinates in $\mathbb{R}^{2}$ allows to establish a quaternion Fourier Mellin transform (QFMT) [22]

$$
\mathscr{F}_{Q M}\{h\}(v, k)=\frac{1}{2 \pi} \int_{0}^{\infty} \int_{0}^{2 \pi} r^{-f v} h(r, \theta) e^{-g k \theta} d \theta d r / r, \quad \forall(v, k) \in \mathbb{R} \times \mathbb{Z},
$$

which can characterize 2D shapes rotation, translation and scale invariant, possibly including color encoded in the quaternion valued signal $h: \mathbb{R}^{2} \rightarrow \mathbb{H}$ such that $|h|$ is summable over $\mathbb{R}_{+}^{*} \times \mathbb{S}^{1}$ under the measure $d \theta d r / r, \mathbb{R}^{*}$ the multiplicative group of positive non-zero numbers, and $f, g \in \mathbb{H}$ two $\sqrt{-1}$. The QFMT can be generalized straightforward to a Clifford Fourier Mellin transform applied to signals $h: \mathbb{R}^{2} \rightarrow C l(p, q), p+q=2[18]$, with $f, g \in C l(p, q), p+q=2$.

The spacetime algebra $C l(3,1)$ of Minkowski space with orthonormal vector basis $\left\{\mathbf{e}_{t}, \mathbf{e}_{1}, \mathbf{e}_{2}, \mathbf{e}_{3}\right\},-\mathbf{e}_{t}^{2}=\mathbf{e}_{1}^{2}=\mathbf{e}_{2}^{2}=$ $\mathbf{e}_{3}^{3}$, has three blades $\mathbf{e}_{t}, i_{3}, i_{s t}$ of time vector, unit space volume 3 -vector and unit hyperspace volume 4 -vector, which are isomorphic to Hamilton's three quaternion units

$$
\mathbf{e}_{t}^{2}=-1, \quad i_{3}=\mathbf{e}_{1} \mathbf{e}_{2} \mathbf{e}_{3}=\mathbf{e}_{t}^{*}=\mathbf{e}_{t} i_{3}^{-1}, i_{3}^{2}=-1, \quad i_{s t}=\mathbf{e}_{t} i_{3}, i_{s t}^{2}=-1 .
$$

The $C l(3,1)$ subalgebra with basis $\left\{1, \mathbf{e}_{t}, i_{3}, i_{s t}\right\}$ is therefore isomorphic to quaternions and allows to generalize the two-sided QFT to a volume-time Fourier transform

$$
\mathscr{F}_{V T}\{h\}(\omega)=\int_{\mathbb{R}^{3,1}} e^{-\mathbf{e}_{t} \omega_{t}} h(\mathbf{x}) e^{-\vec{x} \cdot \vec{\omega}} d^{4} \mathbf{x}
$$

with $\mathbf{x}=t \mathbf{e}_{t}+\vec{x} \in \mathbb{R}^{3,1}, \vec{x}=x_{1} \mathbf{e}_{1}+x_{2} \mathbf{e}_{2}+x_{3} \mathbf{e}_{3}, \omega=\omega_{t} \mathbf{e}_{t}+\vec{\omega} \in \mathbb{R}^{3,1}, \vec{\omega}=\omega_{1} \mathbf{e}_{1}+\omega_{2} \mathbf{e}_{2}+\omega_{3} \mathbf{e}_{3}$. The split (6) with $f=\mathbf{e}_{t}, g=i_{3}=\mathbf{e}_{t}^{*}$ becomes the spacetime split of special relativity

$$
h_{ \pm}=\frac{1}{2}\left(1 \pm \mathbf{e}_{t} h \mathbf{e}_{t}^{*}\right) .
$$




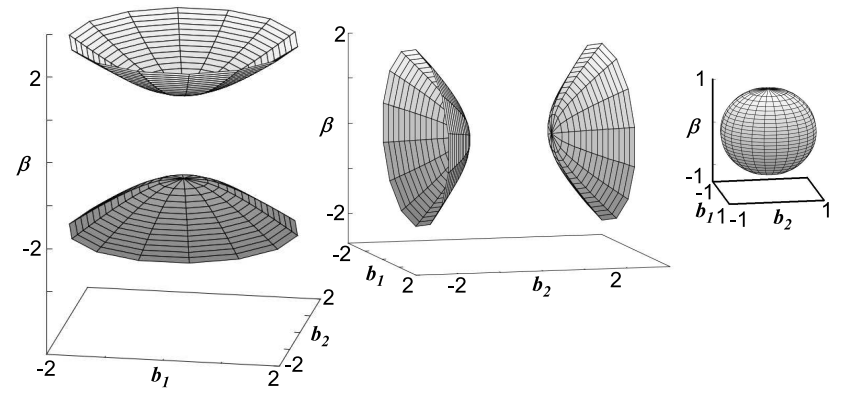

FIGURE 1. Manifolds [21] of square roots $f$ of -1 in $C l(2,0)$ (left), $C l(1,1)$ (center), and $C l(0,2) \cong \mathbb{H}$ (right). The square roots are $f=\alpha+b_{1} e_{1}+b_{2} e_{2}+\beta e_{12}$, with $\alpha, b_{1}, b_{2}, \beta \in \mathbb{R}, \alpha=0$, and $\beta^{2}=b_{1}^{2} e_{2}^{2}+b_{2}^{2} e_{1}^{2}+e_{1}^{2} e_{2}^{2}$.

It is most interesting to observe, that the volume-time Fourier transform can indeed be applied to multivector signal functions valued in the whole spacetime algebra $h: \mathbb{R}^{3,1} \rightarrow C l(3,1)$ without changing its form $[14,17]$

$$
\mathscr{F}_{S T}\{h\}(\omega)=\int_{\mathbb{R}^{3,1}} e^{-\mathbf{e}_{t} \omega_{t}} h(\mathbf{x}) e^{-i_{3} \vec{x} \cdot \vec{\omega}} d^{4} \mathbf{x} .
$$

The split (11) applied to spacetime Fourier transform (12) leads to a multivector wavepacket analysis

$$
\mathscr{F}_{S T}\{h\}(\omega)=\int_{\mathbb{R}^{3,1}} h_{+}(\mathbf{x}) e^{-i_{3}\left(\vec{x} \cdot \vec{\omega}-t \omega_{t}\right)} d^{4} \mathbf{x}+\int_{\mathbb{R}^{3,1}} h_{-}(\mathbf{x}) e^{-i_{3}\left(\vec{x} \cdot \vec{\omega}+t \omega_{t}\right)} d^{4} \mathbf{x},
$$

in terms of right and left propagating spacetime multivector wave packets.

Finally, we turn to one-sided CFTs, which are obtained by setting the phase function $u=0$ in (4). A recent discrete spinor CFT used for edge and texture detection is given in [1], where the signal is represented as a spinor and the $\sqrt{-1}$ is a local tangent bivector $B \in C l(3,0)$ to the image intensity surface ( $\mathbf{e}_{3}$ is the intensity axis). [15]

$$
\mathscr{F}_{P S}\{h\}(\omega)=\int_{\mathbb{R}^{n}} h(\mathbf{x}) e^{-i_{n} \mathbf{x} \cdot \omega} d^{n} \mathbf{x}, \quad i_{n}=\mathbf{e}_{1} \mathbf{e}_{2} \ldots \mathbf{e}_{n}, \quad n=2,3(\bmod 4),
$$

where $h: \mathbb{R}^{n} \rightarrow C l(n, 0)$, and $\left\{\mathbf{e}_{1}, \mathbf{e}_{2}, \ldots, \mathbf{e}_{n}\right\}$ is the orthonormal basis of $\mathbb{R}^{n}$. Historically the special case of (14), $n=3$, was already introduced in 1990 [23] for the processing of electromagnetic fields. This same transform was later applied [12] to two-dimensional images embedded in $C l(3,0)$ to yield a two-dimensional analytic signal, and in image structure processing. Moreover, the pseudoscalar $C F T$ (14), $n=3$, was successfully applied to three-dimensional vector field processing in $[7,6]$ with vector signal convolution based on Clifford's full geometric product of vectors. The theory of the transform has been thoroughly studied in [15].

For embedding one-dimensional signals in $\mathbb{R}^{2},[12]$ considered in (14) the special case of $n=2$, and in $[7,6]$ this was also applied to the processing of two-dimensional vector fields.

Recent applications of (14) with $n=2,3$, to geographic information systems and climate data can be found in $[29,28,24]$.

Soli deo gloria. I do thank my dear family, T. Simos, W. Sprössig and K. Gürlebeck.

\section{REFERENCES}

1. T. Batard, M. Berthier, CliffordâĂŞFourier Transf. and Spinor Repr. of Images, in: E. Hitzer, S.J. Sangwine (eds.), "Quaternion and Clifford Fourier Transf. and Wavelets", TIM 27, Birkhauser, Basel, 2013, 177-195.

2. F. Brackx, et al, History of Quaternion and Clifford-Fourier Transf., in: E. Hitzer, S.J. Sangwine (eds.), "Quaternion and Clifford Fourier Transf. and Wavelets", TIM 27, Birkhauser, Basel, 2013, xi-xxvii.

3. T. Buelow, Hypercomplex Spectral Signal Repr. for the Proc. and Analysis of Images, PhD thesis, Univ. of Kiel, Germany, Inst. fuer Informatik und Prakt. Math., Aug. 1999.

4. T. Buelow, et al, Non-comm. Hypercomplex Fourier Transf. of Multidim. Signals, in G. Sommer (ed.), "Geom. Comp. with Cliff. Algebras", Springer 2001, 187-207. 


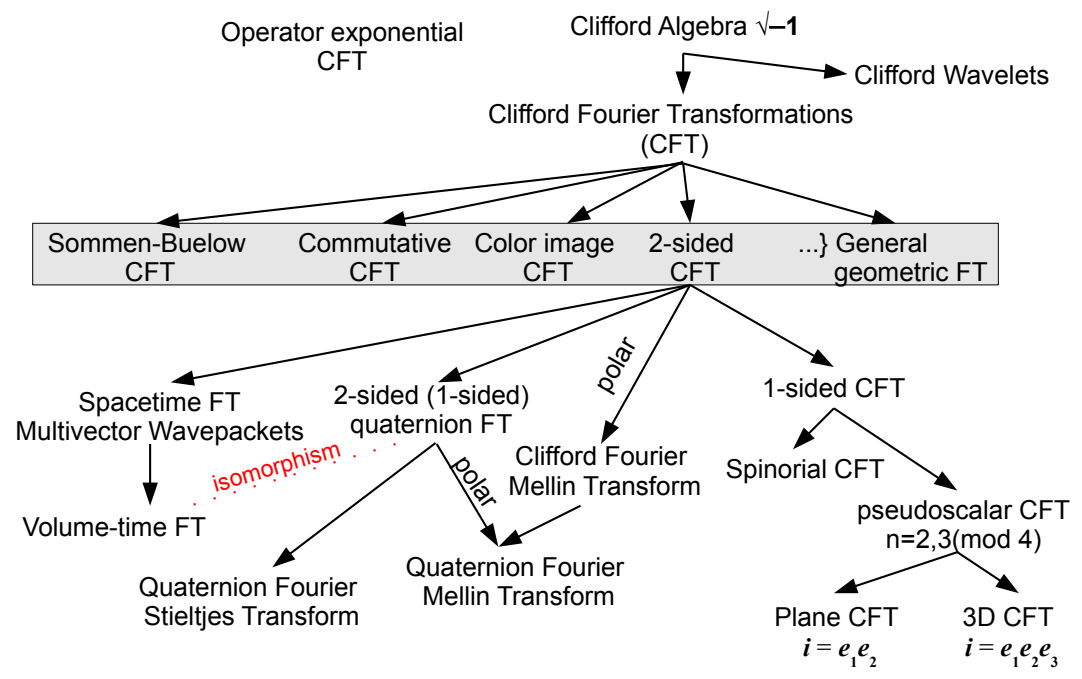

FIGURE 2. Family tree of Clifford Fourier transformations.

5. R. Bujack, et al, A General Geom. Fourier Transf., in: E. Hitzer, S.J. Sangwine (eds.), "Quaternion and Clifford Fourier Transf. and Wavelets", TIM 27, Birkhauser, Basel, 2013, 155-176.

6. J. Ebling, G. Scheuermann, Clifford convolution and pattern matching on vector fields, In Proc. IEEE Vis., 3, IEEE Computer Society, Los Alamitos, 2003. 193-200,

7. J. Ebling, G. Scheuermann, Cliff. Four. transf. on vector fields, IEEE Trans. on Vis. and Comp. Graph., 11(4), (2005), 469-479.

8. T. A. Ell, Quaternionic Fourier Transform for Analysis of Two-dimensional Linear Time-Invariant Partial Differential Systems. in Proceedings of the 32nd IEEE Conference on Decision and Control, December 15-17, 2 (1993), 1830-1841.

9. T.A. Ell, S.J. Sangwine, Hypercomplex Fourier transforms of color images, IEEE Trans. Image Process., 16(1) (2007), 22-35.

10. R. R. Ernst, et al, Princ. of NMR in One and Two Dim., Int. Ser. of Monogr. on Chem., Oxford Univ. Press, 1987.

11. M. Felsberg, et al, Comm. Hypercomplex Fourier Transf. of Multidim. Signals, in G. Sommer (ed.), "Geom. Comp. with Cliff. Algebras", Springer 2001, 209-229.

12. M. Felsberg, Low-Level Img. Proc. with the Struct. Multivec., PhD thesis, Univ. of Kiel, Inst. fuer Inf. \& Prakt. Math., 2002.

13. S. Georgiev, J. Morais, Bochner's Theorems in the Framework of Quaternion Analysis in: E. Hitzer, S.J. Sangwine (eds.), "Quaternion and Clifford Fourier Transf. and Wavelets", TIM 27, Birkhauser, Basel, 2013, 85-104.

14. E. Hitzer, Quaternion Fourier Transform on Quaternion Fields and Generalizations, AACA, 17 (2007), $497-517$.

15. E. Hitzer, B. Mawardi, Clifford Fourier Transf. on Multivector Fields and Unc. Princ. for Dim. $n=2(\bmod 4)$ and $n=3(\bmod$ 4), P. Angles (ed.), AACA, 18(S3,4), (2008), 715-736.

16. E. Hitzer, Cliff. (Geom.) Alg. Wavel. Transf., in V. Skala, D. Hildenbrand (eds.), Proc. GraVisMa 2009, Plzen, 2009, 94-101.

17. E. Hitzer, Directional Uncertainty Principle for Quaternion Fourier Transforms, AACA, 20(2) (2010), 271-284.

18. E. Hitzer, Clifford Fourier-Mellin transform with two real square roots of -1 in $C l(p, q), p+q=2$, 9th ICNPAA 2012, AIP Conf. Proc., 1493, (2012), 480-485.

19. E. Hitzer, Two-sided Clifford Fourier transf. with two square roots of -1 in $\mathrm{Cl}(p, q)$, in M. Berthier, et al (eds.) elect. Proc. of AGACSE 2012, La Rochelle, France, 2012. http: / / arxiv . org/abs/1306.2092

20. E. Hitzer, S. J. Sangwine, The Orthogonal 2D Planes Split of Quaternions and Steerable Quaternion Fourier Transf., in: E. Hitzer, S.J. Sangwine (eds.), "Quaternion and Clifford Fourier Transf. and Wavelets", TIM 27, Birkhauser, Basel, 2013, $15-39$.

21. E. Hitzer, et al, Square roots of -1 in real Clifford algebras, in: E. Hitzer, S.J. Sangwine (eds.), "Quaternion and Clifford Fourier Transf. and Wavelets", TIM 27, Birkhauser, Basel, 2013, 123-153.

22. E. Hitzer, Quaternionic Fourier-Mellin Transf., in T. Sugawa (ed.), Proc. of ICFIDCAA 2011, Hiroshima, Japan, Tohoku Univ. Press, Sendai (2013), ii, 123-131.

23. B. Jancewicz. Trivector Fourier transf. and electromag. Field, J. of Math. Phys., 31(8), (1990), 1847-1852.

24. Linwang Yuan, et al, Geom. Alg. for Multidim.-Unified Geogr. Inf. System, AACA, 23 (2013), 497-518.

25. B. Mawardi, E. Hitzer, Clifford Algebra Cl(3,0)-valued Wavelet Transf., Clifford Wavelet Uncertainty Inequality and Clifford Gabor Wavelets, Int. J. of Wavelets, Multiresolution and Inf. Proc., 5(6) (2007), 997-1019.

26. J. Mennesson, et al, Color Obj. Recogn. Based on a Clifford Fourier Transf., in L. Dorst, J. Lasenby, "Guide to Geom. Algebra in Pract.", Springer 2011, 175-191.

27. S. J. Sangwine, Fourier transf. of color images using quat., or hyperc., numbers, El. Lett., 32(21) (1996), 1979-1980.

28. Yuan Linwang, et al, Pattern Forced Geophys. Vec. Field Segm. based on Clifford FFT, To appear in Computer \& Geoscience.

29. Yu Zhaoyuan, et al, Clifford Algebra for Geophys. Vector Fields, To appear in Nonlinear Process in Geophysics.

E. Hitzer, Extending Fourier transformations to Hamilton's quaternions and Clifford's geometric algebras, In T. Simos, G. Psihoyios and C. Tsitouras (eds.), Numerical Analysis and Applied Mathematics ICNAAM 2013, AIP Conf. Proc. 1558, pp. 529 -532 (2013). DOI: 10.1063/1.4825544. 